\title{
How estrogen fuels breast cancer
}
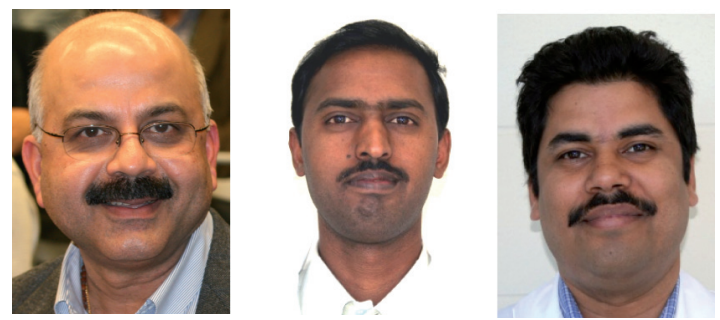

“...sustained exposure of

ER with endogenous or

exogenous estrogen ... is

well established to cause

breast cancer..."

Ajay Rana ${ }^{\dagger 1}$, Velusamy Rangasamy' \& Rajakishore Mishra

'Department of Molecular Pharmacology \& Therapeutics, Loyola University Medical Center, Building 102

2160 South First Avenue, Maywood, IL 60153, USA

†Author for correspondence: Tel.: +1 7082165761 = Fax: +1 7082166596 = arana@|umc.edu

Despite significant progress made to diagnose and treat breast cancer, it still remains the second largest killer in women, just after lung cancer [101]. It is estimated that approximately 190,000 new cases of breast cancer will be diagnosed in USA alone in 2009 [1] and the majority $(\sim 70 \%)$ of them will be estrogen receptor (ER) positive cases. The ER regulates growth, differentiation and homeostasis of the normal mammary gland [2]; however, sustained exposure of ER with endogenous or exogenous estrogen $\left(E_{2}\right)$ is well established to cause breast cancer [3,4]. Sustained activation of ER in breast epithelial cells causes hyperactivation of ER downstream survival pathways, leading to dysregulated growth and thus breast cancer. Therefore, the agents that block either $\mathrm{E}_{2}$ synthesis (i.e., aromatase inhibitors) or antagonize $\mathrm{E}_{2}$ 's action (e.g., tamoxifen and other SERMs) are in clinical use to control the growth of $\mathrm{ER}^{+}$ breast cancer cells. Tamoxifen is certainly the first line of drug therapy for $\mathrm{ER}^{+}$breast cancer [5], whereas aromatase inhibitors have proven highly effective in postmenopausal women with $\mathrm{ER}^{+}$breast cancer [6]. However, these drugs are not effective for long-term use and are known to cause collateral damage to the heart and other organs. In addition, some women are not responsive to hormonal therapy; and will have to undergo chemotherapy or radiotherapy. Therefore, two of the most challenging tasks for $\mathrm{ER}^{+}$breast cancer treatment are the identification of new therapeutic targets for endocrine resistance and the development of predictive biomarkers of endocrine therapy.

The prevailing hypothesis in breast cancer is that estrogen promotes the survival of $\mathrm{ER}^{+}$ breast cancer cells by inhibiting the proapoptotic cellular machinery [7]. It has been demonstrated that ER mediates the downregulation of proapoptotic molecules. However, understanding the detailed network is certainly warranted in finding the primary targets by which ER promotes dysregulated cell growth and could prove beneficial for $\mathrm{ER}^{+}$breast cancer treatment. Recently, we have identified a novel molecule that could serve as a viable target for ER ${ }^{+}$ breast cancer treatment. The role of this molecule, termed Mixed Lineage Kinase 3 (MLK3) in breast cancer pathogenesis, has never been demonstrated before.

“...ER mediates the downregulation of proapoptotic molecules. However, understanding the detail network is certainly warranted in finding the primary targets by which ER promotes dysregulated cell growth and could prove beneficial for $\mathrm{ER}^{+}$breast cancer treatment."

Mixed Lineage Kinase 3 belongs to the family of MAPKKK, termed Mixed Lineage Kinases (MLKs). The MLK family members are characterized by the presence of signature sequences of serine/threonine and tyrosine kinases within their catalytic domain $[8,9]$. Previous work from our laboratory has shown that MLK3 activates the c-Jun NH2-terminal kinase (JNK) via activation of its upstream kinase SAPK/ERK kinase 1 (SEK1/MKK4) [10]. Furthermore, we also demonstrated that MLK3 was inhibited directly by protein kinase $\mathrm{B}$ (PKB/AKT) [11], a survival protein kinase, which is universally overexpressed in most breast cancer cells and mediates the hormonal or cytotoxic agents' resistance [12]. Since activation of MLK3 causes cell death in neuronal and non-neuronal cells $[11,13]$ and activation of AKT promotes survival, including in breast and other cells, it was tempting to conceive that MLK3 or other MLKs could play a significant role in breast cancer pathogenesis.

We measured MLK3 kinase activities in primary human breast tumors and matching normal breast tissues. Interestingly, the

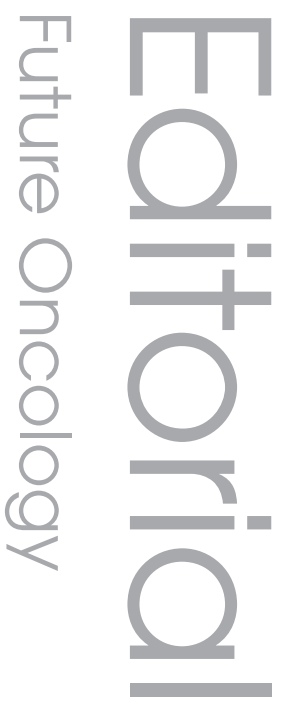

\section{Keywords}

- breast cancer $=$ cytotoxic

drug $=$ estrogen

- estrogen receptor

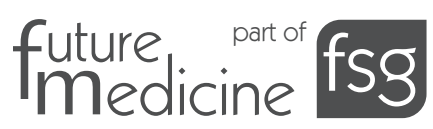


MLK3 kinase activity was significantly higher in some tumors compared with others. Since ER and progesterone receptor (PR) both play a central role in breast cancer pathogenesis, the ER and PR status were also determined in these tumors. Surprisingly, these results revealed that MLK3 kinase activity was approximately five-fold higher, exclusively in the ER tumors compared with $\mathrm{ER}^{+}$[14]. These results certainly suggested to us that MLK3 is most probably negatively regulated by $\mathrm{E}_{2}$. Indeed in $\mathrm{ER}^{+}$human breast cancer cell line, MCF7, $\mathrm{E}_{2}$ inhibited MLK3 kinase activities but not in ER- $\mathrm{SkBr} 3$ cells. Furthermore, $\mathrm{E}_{2}$ action was completely blocked by $\mathrm{E}_{2}$ antagonist, ICI 182,780. As AKT is overexpressed in most breast cancer cells and promotes cell survival, it was interesting to observe that $\mathrm{E}_{2}$-activated AKT, blocked MLK3 kinase activity and AKT or PI3K-specific inhibitors, or AKT-specific siRNA, blocked $\mathrm{E}_{2}$-induced inhibition of MLK3.

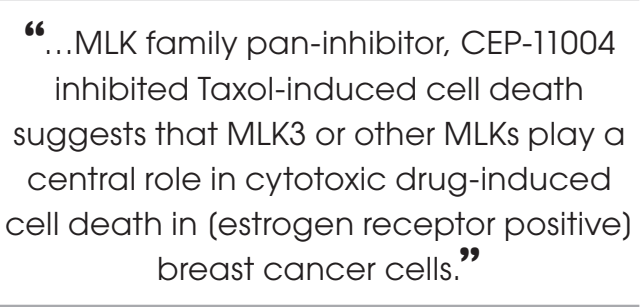

Taxanes, a class of drug that targets the microtubules are often used to treat breast cancer. Taxol, a taxane, has been demonstrated to cause cell death in MCF7 cells and therefore our result that MLK family paninhibitor, CEP-11004 inhibited taxol-induced cell death suggests that MLK3 or other MLKs play a central role in cytotoxic drug-induced cell death in $\mathrm{ER}^{+}$breast cancer cells [14]. The interesting part of our observation was that MLK3 kinase activity was significantly elevated in $\mathrm{ER}^{-}$breast tumors compared to $\mathrm{ER}^{+}$ ones. Furthermore, the fact that ER- tumors are more aggressive compared to $\mathrm{ER}^{+}$tumors raises an important question, how MLK3 activation promotes cell death in $\mathrm{ER}^{+}$tumors? This is certainly a most challenging question and is the primary focus of our laboratory to dissect the role of MLK3 and other MLKs in ER- breast tumors.

\begin{tabular}{c}
\hline "It is already reported that \\
ceramides inhibits AKT; therefore it \\
seems likely that agents such as \\
ceramides or any small molecule, \\
which could inhibit AKT and at the same \\
time activate MLK3 might prove \\
beneficial to promote cell death in \\
(estrogen receptor positive) breast \\
cancer cells."
\end{tabular}

It is interesting to note that previously, we have demonstrated that the bioactive lipids, ceramides functions as an agonist of MLK3 [15]. It is already reported that ceramides inhibits AKT; therefore, it appears likely that agents such as ceramides or any small molecule, which could inhibit AKT and at the same time activate MLK3 might prove beneficial to promote cell death in $\mathrm{ER}^{+}$breast cancer cells. Our results certainly open up a new area of research for any future therapeutic intervention by targeting MLK3, and perhaps other MLK family member(s) for $\mathrm{ER}^{+}$breast cancer treatment.

\section{Financial \& competing interests disclosure The authors have no relevant affiliations or financial involvement with any organization or entity with a financial interest in or financial conflict with the sub- ject matter or materials discussed in the manuscript. This includes employment, consultancies, honoraria, stock ownership or options, expert testimony, grants or patents received or pending, or royalties. \\ No writing assistance was utilized in the production of this manuscript.}

\section{Bibliography}

1. Irvin WJ Jr, Carey LA: What is triplenegative breast cancer? Eur. J. Cancer 44, 2799-2805 (2008).

2. Edwards DP: Regulation of signal transduction pathways by estrogen and progesterone. Annu. Rev. Physiol. 67, 335-376 (2005).

3. Colditz GA: Relationship between estrogen levels, use of hormone replacement therapy, and breast cancer. J. Natl Cancer Inst. 90, 814-823 (1998).
4. Hankinson SE, Colditz GA, Willett WC: Towards an integrated model for breast cancer etiology: the lifelong interplay of genes, lifestyle, and hormones. Breast Cancer Res. 6, 213-218 (2004).

5. Jensen EV, Jordan VC: The estrogen receptor: a model for molecular medicine. Clin. Cancer Res. 9, 1980-1989 (2003).

6. Iwase $\mathrm{H}$ : Current topics and perspectives on the use of aromatase inhibitors in the treatment of breast cancer. Breast Cancer 15, 278-290 (2008).
7. Gompel A, Somai S, Chaouat M et al.: Hormonal regulation of apoptosis in breast cells and tissues. Steroids 65, 593-598 (2000).

8. Gallo KA and Johnson GL: Signalling: mixed-lineage kinase control of JNK and p38 MAPK pathways. Nat. Rev. Mol. Cell Biol. 3, 663-672 (2002).

9. Wang LH, Besirli CG, Johnson EM Jr: Mixed-lineage kinases: a target for the prevention of neurodegeneration. Annu. Rev. Pharmacol. Toxicol. 44, 451-474 (2004). 
10. Rana A, Gallo K, Godowski P et al.: The mixed lineage kinase SPRK phosphorylates and activates the stress-activated protein kinase activator, SEK-1. J. Biol. Chem. 271, 19025-19028 (1996).

11. Barthwal MK, Sathyanarayana P, Kundu CN et al:: Negative regulation of mixed lineage kinase 3 by protein kinase B/AKT leads to cell survival. J. Biol. Chem. 278, 3897-3902 (2003).

12. Riggins RB, Bouton AH, Liu MC, Clarke R: Antiestrogens, aromatase inhibitors, and apoptosis in breast cancer. Vitam. Horm. 71, 201-237 (2005).
13. Xu Z, Maroney AC, Dobrzanski P, Kukekov NV, Greene LA: The MLK family mediates c-Jun N-terminal kinase activation in neuronal apoptosis. Mol. Cell Biol. 21, 4713-4724 (2001).

14. Rangasamy V, Mishra R, Mehrotra S et al.: Estrogen suppresses MLK3-mediated apoptosis sensitivity in $\mathrm{ER}^{+}$breast cancer cells. Cancer Res. 70, 1731-1740 (2010).

15. Sathyanarayana P, Barthwal MK, Kundu CN et al.: Activation of the Drosophila MLK by ceramide reveals TNF- $\alpha$ and ceramide as agonists of mammalian MLK3. Mol. Cell. 10, 1527-1533 (2002).

\section{Website}

101. Breast Cancer Facts, American Cancer Society www.cancer.org/Research/ CancerFactsFigures/

BreastCancerFactsFigures/breast-cancer-facts-figures-2009-2010 